\title{
A Stepwise Approach for Preventing Suicide by Lethal Poisoning
}

\author{
Jasmine E. Carpenter, PharmD, BCPP; Tiffany Lee, PharmD; Elizabeth Greene, MD; and Eileen Holovac, PharmD
}

Background: Suicide is a global phenomenon and is the 10th leading cause of death in the US. Veterans are more likely to die by suicide than those in the general population. In 2018 , the suicide rate for all US veterans was 1.5 times higher than the rate for nonveterans, after adjusting for population differences in age and sex. In light of this disparity, suicide prevention is one of the highest priorities for the US Department of Veterans Affairs (VA). One major goal of the VA suicide prevention strategy is to reduce access to lethal means.

Observations: This article will provide information on medications with high lethality and a stepwise approach for how health care providers may limit lethal medications for patients at high risk for suicide. The first step is to determine suicide risk. More than $90 \%$ of those who die by suicide have a psychiatric diagnosis at the time of death. Clinicians can use risk assessment tools, such as the Veterans Health Administration Suicide Prevention Population Risk Identification and Tracking for Exigencies tool. The second step is to identify substances strongly associated with fatalities. According to the American Association of Poison Control Centers, the pharmaceutical classes associated with the largest number of fatalities are stimulants and street drugs, followed by analgesics, cardiovascular agents, antidepressants, antipsychotics, and sedatives/hypnotics. The third step is to consider potential drug-drug interactions, such as the combination of opioids and sedative-hypnotics. Finally, clinicians need to address risks. With high-risk patients it may be preferential to prescribe medications that are less lethal. All patients with a high risk of suicide should receive lethal means counseling.

Conclusions: While firearms continue to be the most lethal means for veteran suicide, intentional poisoning with medications or substances also is a common method for suicide, especially for female veterans. Having knowledge of medications with high lethality and limiting access to these agents can be a successful strategy for reducing suicide deaths.
Jasmine Carpenter is a Mental Health Clinical Pharmacy Specialist; Tiffany Lee is a Geriatric Clinical Pharmacy Specialist; and Elizabeth Green is a Women's Clinic Psychiatrist; all at the Washington Veterans Affairs Medical Center in the District of Columbia. Eileen Holovac is an Oncology Clinical Pharmacy Specialist at the Palo Alto Veterans Affairs Medical Center in California.

Correspondence: Jasmine Carpenter (jasmine.carpenter@va.gov)

Fed Pract. 2021;38(2):62-67. doi:10.12788/fp.0087
$(0$ uicide is a global phenomenon and a worldwide public health concern. ${ }^{1}$ The World Health Organization estimates that $>800,000$ people die by suicide every year. In the US, suicide is the 10th leading cause of death, and on average, 129 Americans die by suicide each day. ${ }^{2}$ In 2018 , the suicide rate for all veterans was 1.5 times higher than the rate for nonveterans, after adjusting for population differences in age and sex. Among female veterans, the rate of suicide was 2.1 times higher than the rate for female nonveterans. ${ }^{3}$

In light of this disparity, suicide prevention is one of the highest priorities for the US Department of Veterans Affairs (VA). In 2018, the VA developed and published the National Strategy for Preventing Veteran Suicide. ${ }^{4}$ One major goal of this strategy is to reduce access to lethal means (ie, firearms, medications, chemicals, or poisons) among veterans at high risk for suicide. Reducing access to lethal means has been found to decrease suicide rates. ${ }^{4,5}$

Drug overdose is a leading method for suicide attempts, es- pecially for female veterans..$^{3,6}$ Although the overall case fatality ratio for overdose is $<2 \%$, drug overdose accounted for $59.4 \%$ of suicide attempts and $13.5 \%$ of deaths by suicide from 2007 to $2014 .{ }^{6,7}$ Within the veteran population, the majority of suicide deaths in 2018 were due to self-inflicted firearm injury for both male and female veterans, followed by poisoning via substances and pharmaceutical agents for female veterans (Figure 1). ${ }^{3}$ Notably, when compared with men, women were more likely to choose drug overdose as a method for suicide. One study found

FIGURE 1 Method of Suicide Among Veterans by Gender, 2018

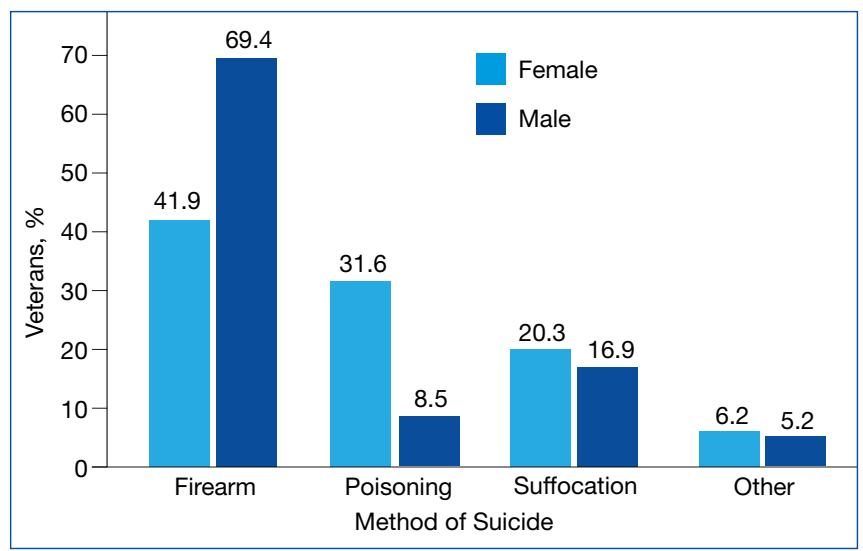


that women aged $<45$ years used drug and poison ingestion in 9 out of 10 suicide attempts. ${ }^{6}$ Since some medications are more lethal than others, interventions to limit the availability of lethal medications may prevent deaths and reduce the severity of suicide attempts. This article will provide a stepwise approach to help clinicians identify and limit lethal medications for patients at high risk for suicide.

\section{STEP 1: DETERMINE SUICIDE RISK}

Although it is impossible to predict with certainty an individual's risk of suicide, several patient characteristics and life circumstances have been identified as risk factors. The strongest predictor of suicide is the presence of psychiatric disease. ${ }^{8}$ More than $90 \%$ of those who have had a death by suicide have a psychiatric diagnosis at the time of death, and suicide rates in those with mental illness are at least 10 times as high as in the general population. ${ }^{9}, 10$ Depression is the leading cause of death by suicide worldwide, followed by substance-related disorders $(22.4 \%)$, personality disorders (11.6\%), schizophrenia (10.6\%), and anxiety/somatoform disorders (6.1\%). . $^{811-13}$

Clinicians also can use various risk assessment tools to identify patients at high risk for suicide. The Veterans Health Administration (VHA) Stratification Tool for Opioid Risk Mitigation (STORM) calculates patients' risk based on data extracted from the electronic health record and is less time intensive, more easily refined, and may be more powerful than standard risk assessment tools because it can be deployed on a large scale..$^{14,15}$ The VHA also developed the Suicide Prevention Population Risk Identification and Tracking for Exigencies (SPPRITE) tool to assist clinicians in tracking patients with current (or recent) high levels of suicide risk. This tool unifies specific patient information gathered from the patient's electronic health record and from other predictive model dashboards (such as STORM).

\section{STEP 2: IDENTIFY SUBSTANCES STRONGLY ASSOCIATED WITH FATALITIES}

According to the American Association of Poison Control Centers (AAPCC), the pharmaceutical classes associated with the largest
TABLE 1 Pharmaceutical Drug Classes Associated With Fatalities from the 2018 Poison Control Annual Report ${ }^{16}$

\begin{tabular}{|c|c|}
\hline Medications & Fatalities, No. \\
\hline Analgesics & 834 \\
\hline Opioids & 589 \\
\hline Acetaminophen & 145 \\
\hline Salicylate & 46 \\
\hline Acetaminophen/diphenhydramine & 12 \\
\hline Stimulants and Street Drugs & 694 \\
\hline Methamphetamine & 321 \\
\hline Heroin & 242 \\
\hline Cocaine & 77 \\
\hline Kratom & 8 \\
\hline Amphetamine & 8 \\
\hline MDMA & 7 \\
\hline THC homologs & 6 \\
\hline Cardiovascular Drugs & 232 \\
\hline CCBs & 116 \\
\hline$\beta$ blockers & 31 \\
\hline Digoxin & 13 \\
\hline Flecainide & 10 \\
\hline Antidepressants & 144 \\
\hline TCAs & 52 \\
\hline Bupropion & 49 \\
\hline Trazodone & 9 \\
\hline Venlafaxine & 8 \\
\hline Sedatives/Hypnotics/Antipsychotics & 97 \\
\hline Benzodiazepines & 41 \\
\hline Quetiapine & 21 \\
\hline Zolpidem & 7 \\
\hline Olanzapine & 6 \\
\hline
\end{tabular}

Abbreviations: BZD, benzodiazepine; CCB, calcium channel blocker; MDMA, methylenedioxymethamphetamine;

THC,tetrahydrocannabinol; TCA, tricyclic antidepressants.

number of fatalities are analgesics, followed by stimulants and street drugs, cardiovascular agents, antidepressants, antipsychotics, and sedatives/hypnotics (Table 1). ${ }^{16}$ Stimulants and street drugs accounted for 694 fatalities of 39,238 single-substance exposures (mortality rate: $1.8 \%$ ). ${ }^{16}$ Drugs of abuse, including cocaine, hallucinogenic amphetamines, heroin, and kratom, have shown an increased trend in use. ${ }^{16}$

In 2018 there were 834 fatalities from 174,269 single-substance exposure to analgesics, which include opioids and acetaminophen, for a mortality rate of $0.5 \%{ }^{16}$ The opioid epidemic is one of the main drivers of the increase in drug overdose deaths in the US. ${ }^{16,17}$ The opioid with the highest drug overdose fatality rate is 
TABLE 2 Mean Mortality Indices per 10,000 Exposures ${ }^{23}$

\begin{tabular}{lc} 
Pharmaceuticals & Mortality Index \\
\hline Acetaminophen & 25.8 \\
\hline Diphenhydramine & 4.8 \\
\hline Tricyclic antidepressants & 40.7 \\
\hline Serotonin-norepinephrine reuptake inhibitor & 7.2 \\
\hline Second-generation antidepressants & \\
Bupropion & 7.5 \\
Venlafaxine & 9.7 \\
Citalopram & 4.2 \\
Sertraline & 0.9 \\
Fluoxetine & 0.8 \\
\hline Atypical antipsychotics & \\
Quetiapine & 7.6 \\
Olanzapine & 6.1 \\
Ziprasidone & 5.6 \\
Risperidone & 2.5 \\
Aripiprazole & 0.0 \\
\hline Mood stabilizers & \\
Lithium & 13.2 \\
Valproic acid & 8.1 \\
Carbamazepine & 5.6 \\
Lamotrigine & 2.2 \\
\hline$\quad$
\end{tabular}

illicitly manufactured fentanyl, which often is combined with other substances, such as heroin, to increase its potency at a low cost. ${ }^{18}$ These combinations also increase the risk of overdose fatality.

Acetaminophen is unique among the top substances associated with fatalities because it is obtained easily without a prescription. An acetaminophen overdose can cause hepatic injury, which may progress to fulminant hepatic failure and death. ${ }^{19}$ The recommended maximum dose of acetaminophen is $4 \mathrm{~g} / \mathrm{d}$ in an adult and 50 to $75 \mathrm{mg} / \mathrm{kg} / \mathrm{d}$ in children. A single acute ingestion of $>7.5 \mathrm{~g}$ in an adult or $150 \mathrm{mg} / \mathrm{kg}$ in children has been considered potentially toxic. ${ }^{19,20}$ The use of combination analgesics that contain both an opioid and acetaminophen can pose an even greater risk due to the potential for respiratory depression and hepatotoxicity.

Cardiovascular drugs accounted for 232 fatalities from 46,499 single-substance exposures (mortality rate: $0.5 \%$ ). ${ }^{16}$ According to the AAPCC, calcium channel blockers (CCB) and $\beta$-blockers accounted for $63 \%$ of overdose deaths by cardiovascular drugs because they can cause severe hy- potension, bradycardia, and hemodynamic collapse. $^{16,21,22}$

In the past, the nondihydropyridine CCBs verapamil and diltiazem were associated with increased overdose fatalities. However, the most recent data show that dihydropyridine CCBs such as amlodipine also have significant risk for lethality. ${ }^{16}$ Metoprolol was associated with more overdose deaths in the past year among $\beta$-blockers. However, caution also should be used with agents such as propranolol and labetalol, which can antagonize sodium channels in overdose and may be associated with a higher risk of mortality than other $\beta$-blockers. ${ }^{22}$

Antidepressants accounted for 144 fatalities from 56,891 single-substance exposures (mortality rate: $0.3 \%) .{ }^{16}$ Nelson and Spyker performed a study to determine the morbidity and mortality index for psychotropic agents based on exposure reports from the National Poison Data system and found that tricyclic antidepressants (TCAs) and monoamine oxidase inhibitors (MAOIs) had the highest morbidity and mortality rates among all drugs used to treat depression. ${ }^{23}$ As a class, TCAs have a mortality index of 40.7 per 10,000 exposures and are associated with higher rates of acidosis, cardiac conduction problems, respiratory depression, and seizures (Table 2). ${ }^{23} \mathrm{Am}$ itriptyline accounted for $39.5 \%$ of deaths from antidepressants. ${ }^{23}$ Among newer antidepressants, citalopram, venlafaxine, and bupropion have been found to be the most hazardous. ${ }^{23}$ Citalopram and venlafaxine have morbidity indices that are 4- to 5-fold higher than sertraline. Adverse events associated with bupropion, venlafaxine, and citalopram, such as seizures, conduction disturbances, hallucinations, and tachycardia contribute to the morbidity and mortality related to these medications (Table 3 ).

Of the atypical antipsychotics, olanzapine, quetiapine, and ziprasidone have the highest mortality rates. ${ }^{23}$ Cardiac conduction problems were more frequent with olanzapine and ziprasidone, and respiratory depression was more frequent with olanzapine and quetiapine. Aripiprazole had the lowest rates of morbidity and mortality. ${ }^{23}$

Of the mood stabilizers, lithium, valproic acid, and carbamazepine have narrow therapeutic indices and, therefore, moderately 
high mortality rates. ${ }^{23}$ Lithium was associated with higher rates of bradycardia, confusion, and renal problems. Valproic acid had relatively high levels of acidosis and coma. Carbamazepine had high rates of coma and the highest rate of nystagmus.

Sedatives and hypnotics accounted for 97 fatalities of 51,495 single-substance exposures (mortality rate $0.2 \%$ ). ${ }^{16}$ Within this category, benzodiazepines (BZDs), particularly alprazolam, clonazepam, and diazepam, were associated with the highest number of overdose deaths. ${ }^{16}$ Although fatalities from singlesubstance exposure to this category are low, it should be noted that BZDs are primarily metabolized by the CYP2C19 and CYP3A4 enzymes. Interactions with other drugs also metabolized by the same CYP enzymes may lead to prolonged effects of BZDs, such as sedation, and respiratory depression, which significantly increase the risk of overdose death. Furthermore, lipophilic BZDs, such as diazepam, can accumulate in the tissue after multiple doses and have impaired clearance in older patients.

\section{STEP 3: CONSIDER POTENTIAL DRUG-DRUG INTERACTIONS}

Suicide attempts involving multiple substances carry increased risk. Only $12.1 \%$ of all fatal overdoses, according to AAPCC, involved single-substance exposure, whereas $56.3 \%$ were attributed to multiple substance exposures. ${ }^{16}$ It is important for clinicians to be aware of and avoid possibly fatal drugdrug interactions, such as the combination of opioids and sedative-hypnotics, like BZDs, which can lead to fatal respiratory depression. Clinicians also should be aware of a patient's history of illicit opioid and alcohol use before prescribing opioids and BZDs. Clinicians can use various online databases to detect potential drug-drug interactions.

\section{STEP 4: ADDRESS RISKS}

If a patient is deemed to be at high risk for suicide, but it is not imminent and the patient will be managed as an outpatient, then it may be preferential to prescribe medications that are less lethal, such as SSRIs, instead of TCAs or MAOIs. If a potentially lethal medication is indicated, such as lithium or clozapine, both of which have been found to reduce suicidal behavior, then
TABLE 3 Serious Adverse Events Associated With Second-Generation Antidepressants ${ }^{23}$

\begin{tabular}{ll} 
Medications & Adverse Events \\
\hline Bupropion & - Highest rate of single and multiple seizures \\
& $\bullet$ Hallucinations \\
\hline Venlafaxine & - Conduction disturbances \\
& - Tachycardia \\
& - Single seizures \\
\hline Citalopram & - Conduction disturbances \\
& - Seizures \\
& - Acidosis \\
& $\bullet$ Electrolyte disturbances
\end{tabular}

TABLE 4 Psychotropics With High Relative Lethality for a 30-Day Supply²6

\begin{tabular}{lc} 
Psychotropics & $\begin{array}{c}\text { Human Equivalent } \\
\text { Dose Relative } \\
\text { Lethality, \% }\end{array}$ \\
\hline Antidepressants & 632.7 \\
Doxepin & 387.5 \\
Amitriptyline & 319.6 \\
Nefazodone & 304.9 \\
Imipramine & 290.6 \\
Desipramine & 289.4 \\
Bupropion & 269.6 \\
Trazadone & 156.3 \\
Levomilnacipran & 133.3 \\
Duloxetine & \\
\hline Mood stabilizers & $1,665.7$ \\
Valproic acid & $1,062.9$ \\
Lithium & 604.9 \\
Lamotrigine & 253.5 \\
Carbamazepine & \\
\hline Antipsychotics & $1,710.4$ \\
Chlorpromazine & 124.0 \\
Clozapine & \\
Quetiapine &
\end{tabular}

dispensing a limited quantity of pills and having more frequent follow-up visits are some ways to lessen risk. ${ }^{24,25}$ A clinical pearl published in Current Psychiatry provided an equation to determine the lethality of a 30-day supply of medications. ${ }^{26}$ This equation uses lethal dose 50 (LD50), which is the dose of a medication that results in the death of $50 \%$ of the animals used in a controlled experiment, and the maximum daily dose of the medication (D) to find the human equivalent dose (HED) relative lethality. The HED relative lethality calculation may help prescribers determine which medications should have a limited quantity dispensed to

$$
H E D \text { relative lethality }(\%)=\frac{310}{L D 50\left(\frac{\mathrm{mg}}{\mathrm{kg}}\right)} \times D(\mathrm{mg})
$$


patients at risk of medication-related suicide. Any value for the HED relative lethality that is $>100 \%$ is considered a lethal dose for humans. Therefore, it would be appropriate to avoid or limit the quantity of medications with a HED relative lethality $>100 \%$. Table 4 lists the psychotropic agents with the highest relative lethality for a 30-day supply. The psychotropic agents with the lowest HED relative lethality are SSRIs: desvenlafaxine, mirtazapine, topiramate, and aripiprazole. ${ }^{26}$

Limiting drugs with a narrow therapeutic index should be considered when aiming to reduce the risk of medication-related suicide. These drugs present a high risk in the event of an overdose. Clinicians can monitor the levels of lithium, clozapine, or TCAs to ensure that a patient is taking the medication as prescribed rather than stockpiling it at home. If the patient is in a monitored setting, such as a partial hospital program or intensive outpatient program, then the medication can be given while under direct observation.

Clinicians should obtain an accurate and detailed medication and illicit drug use history from patients. It also is important to review the prescription drug monitoring program to limit access to potentially lethal combinations of medications. ${ }^{27}$ Clinicians can additionally employ risk mitigation strategies (eg, providing naloxone kits) for patients who are prescribed or abuse opioids.

Finally, all patients with a high risk of suicide should receive lethal means counseling, which involves first determining whether patients have access to lethal means, such as firearms or medications with high lethality, then limiting their access to these lethal means. This includes advising patients and family members to safely dispose medications that are no longer in use and in some cases recommending that a family member keep medications locked and dispense them on a daily basis.

\section{CONCLUSIONS}

Suicide is a major public health concern that affects tens of thousands of Americans annually. Furthermore, veterans are more likely to die by suicide than those in the general population. Firearms continue to be the most lethal means for suicide. However, intentional poisoning with medications or substances also is a common method for suicide, espe- cially in female veterans. Having knowledge of medications with high lethality and limiting access to these agents can be a successful strategy for reducing suicide deaths.

\section{Author disclosures}

The authors report no actual or potential conflicts of interest with regard to this article.

\section{Disclaimer}

The opinions expressed herein are those of the authors and do not necessarily reflect those of Federal Practitioner, Frontline Medical Communications Inc., the US Government, or any of its agencies. This article may discuss unlabeled or investigational use of certain drugs. Please review the complete prescribing information for specific drugs or drug combinations-including indications, contraindications, warnings, and adverse effectsbefore administering pharmacologic therapy to patients.

\section{References}

1. World Health Organization. Preventing suicide: a global imperative. Published 2014. Accessed January 16,2021 . https://apps.who.int/iris/bitstream /handle/10665/131056/9789241564779_eng.pdf

2. Centers for Disease Control and Prevention, National Center for Injury Prevention and Control, Division of Violence Prevention. National Violent Death Reporting System (NVDRS). Updated November 7, 2019. Accessed January 7,2021 . https://www.cdc.gov/violenceprevention /datasources/nvdrs/

3. US Department of Veteran Affairs, Office of Mental Health and Suicide Prevention. 2020 national veteran suicide prevention annual report. Accessed January 16, 2021. https:// www.mentalhealth.va.gov/docs/data-sheets/2020/2020 - National-Veteran-Suicide-Prevention-Annual -Report-11-2020-508.pdf

4. US Department of Veteran Affairs. National strategy for preventing veteran suicide 2018-2028. Accessed January 7,2021 . https://www.mentalhealth.va.gov/suicide prevention/docs/Office-of-Mental-Health-and-Suicide -Prevention-National-Strategy-for-Preventing-Veterans -Suicide.pdf

5. Zalsman G, Hawton K, Wasserman D, et al. Suicide prevention strategies revisited: 10-year systematic review. Lancet Psychiatry. 2016;3(7):646-659. doi:10.1016/S2215-0366(16)30030-X

6. Spicer RS, Miller TR. Suicide acts in 8 states: incidence and case fatality rates by demographics and method. Am J Public Health. 2000;90(12):1885-1891. doi:10.2105/ajph.90.12.1885

7. Conner A, Azrael D, Miller M. Suicide Case-Fatality Rates in the United States, 2007 to 2014: A Nationwide Population-Based Study. Ann Intern Med. 2019;171(12):885-895. doi:10.7326/M19-1324

8. Bertolote JM, Fleischmann A. Suicide and psychiatric diagnosis: a worldwide perspective. World Psychiatry. 2002;1(3):181-185.

9. Hall RC, Platt DE, Hall RC. Suicide risk assessment: a review of risk factors for suicide in 100 patients who made severe suicide attempts. Evaluation of suicide risk in a time of managed care. Psychosomatics. 1999;40(1):18-27. doi:10.1016/S0033-3182(99)71267-3

10. Bachmann S. Epidemiology of Suicide and the Psychiatric Perspective. Int J Environ Res Public Health. 2018;15(7):1425. Published 2018 Jul 6. doi:10.3390/ijerph15071425

11. Hoertel N, Franco S, Wall MM, et al. Mental disorders and risk of suicide attempt: a national prospective study. Mol Psychiatry. 2015;20(6):718-726. doi:10.1038/mp.2015.19

12. Ferrari AJ, Charlson FJ, Norman RE, et al. Burden of depressive disorders by country, sex, age, and year: findings from the global burden of disease study 2010. PLoS Med. 2013;10(11):e1001547. doi:10.1371/journal.pmed.1001547 
13. World Health Organization. Mental health atlas. Accessed January 7,2021 . https://apps.who.int/iris/bitstream /handle/10665/178879/9789241565011_eng.pdf

14. Velupillai S, Hadlaczky G, Baca-Garcia E, et al. Risk assessment tools and data-driven approaches for predicting and preventing suicidal behavior. Front Psychiatry. 2019;10:36. Published 2019 Feb 13. doi:10.3389/ fpsyt.2019.00036

15. Oliva EM, Bowe T, Tavakoli S, et al. Development and applications of the Veterans Health Administration's Stratification Tool for Opioid Risk Mitigation (STORM) to improve opioid safety and prevent overdose and suicide. Psychol Serv. 2017;14(1):34-49. doi:10.1037/ser0000099

16. Gummin DD, Mowry JB, Spyker DA, et al. 2018 Annual Report of the American Association of Poison Control Centers' National Poison Data System (NPDS): 36th Annual Report [published correction appears in Clin Toxicol (Phila). 2019 Dec;57(12):e1]. Clin Toxicol (Phila). 2019;57(12):12201413. doi: $10.1080 / 15563650.2019 .1677022$

17. Hedegaard H, Miniño AM, Warner M. Drug overdose deaths in the United States, 1999-2018. NCHS Data Brief. 2020(356).

18. Kuczyńska K, Grzonkowski P, Kacprzak Ł, Zawilska JB. Abuse of fentanyl: An emerging problem to face. Forensic Sci Int. 2018;289:207-214. doi:10.1016/j. forsciint.2018.05.042

19. Hodgman MJ, Garrard AR. A review of acetaminophen poisoning. Crit Care Clin. 2012;28(4):499-516. doi:10.1016/j.ccc.2012.07.006

20. Chiew AL, Gluud C, Brok J, Buckley NA. Interventions for paracetamol (acetaminophen) overdose. Cochrane Database Syst Rev. 2018;2(2):CD003328. Published 2018 Feb 23. doi:10.1002/14651858.CD003328.pub3

21. Graudins A, Lee HM, Druda D. Calcium channel antagonist and beta-blocker overdose: antidotes and adjunct therapies. Br J Clin Pharmacol. 2016;81(3):453-461. doi:10.1111/bcp.12763

22. DeWitt CR, Waksman JC. Pharmacology, pathophysiology and management of calcium channel blocker and beta-blocker toxicity. Toxicol Rev. 2004;23(4):223-238. doi:10.2165/00139709-200423040-00003

23. Nelson JC, Spyker DA. Morbidity and mortality associated with medications used in the treatment of depression: an analysis of cases reported to U.S. Poison Control Centers, 2000-2014. Am J Psychiatry. 2017;174(5):438-450. doi:10.1176/appi.ajp.2016.16050523

24. Sarai SK, Mekala HM, Lippmann S. Lithium suicide prevention: a brief review and reminder. Innov Clin Neurosci. 2018;15(11-12):30-32.

25. Meltzer HY, Alphs L, Green Al, et al. Clozapine treatment for suicidality in schizophrenia: International Suicide Prevention Trial (InterSePT) [published correction appears in Arch Gen Psychiatry. 2003 Jul;60(7):735]. Arch Gen Psychiatry. 2003;60(1):82-91. doi:10.1001/archpsyc.60.1.82

26. Giurca D. Decreasing suicide risk with math. Curr Psychiatry. 2018;17(2):57-61.

27. Malte CA, Berger D, Saxon AJ, et al. Electronic medical record alert associated with reduced opioid and benzodiazepine coprescribing in high-risk veteran patients. Med Care. 2018;56(2):171-178. doi:10.1097/MLR.0000000000000861 